\title{
The emergence of systematicity: how environmental and communicative factors shape a novel communication system
}

Jonas Nölle ${ }^{* 1,3}$, Marlene Staib ${ }^{3}$, Riccardo Fusaroli ${ }^{2,3}$, and Kristian Tylén ${ }^{2,3}$

*Corresponding author at: The University of Edinburgh, Room 1.15, Dugald Stewart Building,

3 Charles Street, Edinburgh EH8 9AD, Scotland, United Kingdom

E-mail addresses: j.nolle@sms.ed.ac.uk (J. Nölle), mvs@cc.au.dk (M. Staib),

fusaroli@cc.au.dk (R. Fusaroli), kristian@cc.au.dk (K. Tylén)

${ }^{1}$ Centre for Language Evolution, The University of Edinburgh, Dugald Stewart Building,

3 Charles Street, Edinburgh EH8 9AD, Scotland, United Kingdom

${ }^{2}$ School of Communication and Culture, Aarhus University, Jens Chr. Skous Vej 2, 8000 Aarhus, Denmark

${ }^{3}$ The Interacting Minds Centre, Aarhus University, Jens Chr. Skous Vej 2, 8000 Aarhus, Denmark 


\section{Abstract}

Where does linguistic structure come from? We suggest that systematicity in language evolves adaptively in response to environmental and contextual affordances associated with the practice of communication itself. In two experiments, we used a silent gesture referential game paradigm to investigate environmental and social factors promoting the propagation of systematicity in a novel communication system. We found that structure in the emerging communication systems evolve contingent on structural properties of the environment. More specifically, interlocutors spontaneously relied on structural features of the referent stimuli they communicated about to motivate systematic aspects of the evolving communication system even when idiosyncratic iconic strategies were equally afforded. Furthermore, we found systematicity to be promoted by the nature of the referent environment. When the referent environment was open and unstable, analytic systematic strategies were more likely to emerge compared to stimulus environments with a closed set of referents. Lastly, we found that displacement of communication promoted systematicity. That is, when interlocutors had to communicate about items not immediately present in the moment of communication, they were more likely to evolve systematic solutions, supposedly due to working memory advantages. Together, our findings provide experimental evidence for the idea that linguistic structure evolves adaptively from contextually situated language use.

Keywords: systematicity, iconicity, language evolution, experimental semiotics, silent gesture, interaction

\section{Introduction}

Systematicity permeates language at all levels. Most languages, for instance, show consistent constituent orders (e.g. Subject-Object-Verb vs. Subject-Verb-Object), compositionality (e.g. systematic marking of tense, gender, case, number etc.), semantic 
roles or even systematic sound-meaning mappings as in phonaestheme clusters such as glimmer, glare, glisten (Bergen, 2004; Monaghan, Shillcock, Christiansen, \& Kirby, 2014). Systematicity thus refers to statistical relationships between forms that relate in their meanings, ultimately constituting 'categories' on the form side. But where does systematicity come from? What are the cognitive and communicative factors that promote the persuasive propagation of systematicity across almost all aspects of linguistic structure?

Controversies exist in the language sciences concerning the foundations of linguistic structure. Some theories favour biological explanations grounding linguistic structure in innate genetic code (Chomsky, 1986; Hauser, Chomsky, \& Fitch, 2002; Pinker, 1994). Another prevalent suggestion is that systematicity emerges in response to internal, cognitive biases that get amplified through transmission and learning in processes of cultural evolution (Brighton, 2002; Christiansen \& Chater, 2008; Kirby, Cornish, \& Smith, 2008; Kirby, Griffiths, \& Smith, 2014; Kirby, Tamariz, Cornish, \& Smith, 2015; Smith, Brighton, \& Kirby, 2003). Using the 'iterated learning paradigm', Kirby and colleagues have investigated how sign systems become increasingly ordered, compressible and easier to learn and process, as they are transmitted from one generation to another, due to subtle unintended distortions as signs passes through cognitive bottlenecks and biases of language learners (Kirby et al., 2008). However, it is unclear where this human propensity for systematicity comes from and how specific features are selected and become expressed in systematic categories.

In this paper, we explore the circumstances under which systematic strategies evolve in communicative interaction when referents can also potentially be disambiguated using idiosyncratic, holistic signs. By "circumstances", we mean factors that pertain to aspects of the referent environment and communicative situation. The idea that linguistic structure is promoted by a number of external, contextual factors has recently been coined the Linguistic Niche Hypothesis (Lupyan \& Dale, 2010). In particular, we suggest that systematicity in language emerges adaptively in response to environmental and social factors associated with the situated interactive practice of communication itself (rather than individual learning, 
Tylén, Fusaroli, Bundgaard, \& Østergaard, 2013). That is, linguistic structure is motivated by, and evolves contingent on, structural properties of the physical and social environment. Recent studies provide cross-sectional/correlational evidence for the idea that linguistic structure is contingent on environmental factors, thus suggesting that languages evolve adaptively to meet ecological affordances. Examples include climate influencing the lexicon (Lindsey \& Brown, 2002; Regier, Carstensen, \& Kemp, 2016) or sound systems (Everett, Blasi, \& Roberts, 2015, 2016), subtle genetic or anatomic biases guiding variation (Dediu, Janssen, \& Moisik, 2017; Dediu \& Ladd, 2007), as well as social factors such as number of speakers that has been found to affect morphological complexity (Dale \& Lupyan, 2010; Lupyan \& Dale, 2016).

In this study, we take an experimental approach to address the question whether there are specific environmental circumstances under which systematic categories and signs are more adaptive and thus more likely to evolve in competition with non-systematic strategies that might also provide viable solutions. We first define what distinguishes systematic signs from idiosyncratic signs and then individuate three complementary environmental factors hypothesized to promote systematicity in an evolving communication system: i) inherent structure of the referent scenes, ii) instability/openness of the referent environment, and iii) displacement of the communicative environment from the referential scene

\subsection{Functional pressures for systematicity, iconicity, and arbitrariness}

Systematicity contrasts with other referential principles such as iconicity and arbitrariness that describe the relation between single signs and their meanings. De Saussure (1959) famously argued the defining trait of language to be 'arbitrariness of the linguistic sign' and thus inaugurated a widely endorsed linguistic tradition relegating non-arbitrariness to the role of a rare and peculiar phenomenon to be mostly ignored. However, recent work has highlighted subtle motivations underlying linguistic structure (Kirby, Dowman, \& Griffiths, 2007; Lupyan \& Dale, 2016). Examples include studies of ideophones, sound symbolism 
and systematicity (Dingemanse, 2012; Dingemanse, Blasi, Lupyan, Christiansen, \& Monaghan, 2015; Monaghan, Mattock, \& Walker, 2012). A general tendency in this literature has been to subsume iconicity and systematicity under 'non-arbitrary forms' as opposed to 'arbitrary forms' (e.g. Monaghan et al., 2014). However, iconicity and systematicity could in fact be argued to be the expression of diverse adaptive pressures (Dingemanse et al., 2015).

Iconic signs are motivated in that there is a resemblance between their form and meaning (Peirce, 1931). Iconicity has thus been suggested to play a prominent role in the grounding of communication systems as mappings between form and embodied experience on both phylo- and ontogenetic time scales (Fay, Ellison, \& Garrod, 2014; Harnad, 1990; Perniss, Thompson, \& Vigliocco, 2010). In language acquisition, iconic sound-referent mappings seem to facilitate early word-learning (Imai \& Kita, 2014; Imai, Kita, Nagumo, \& Okada, 2008; Perlman, Fusaroli, Fein, \& Naigles, 2017; Perry, Perlman, \& Lupyan, 2015) as well as novel word learning in adults (Bergen, 2004; Lockwood, Dingemanse, \& Hagoort, 2016). In addition, experimental lab studies of emergent communication systems indicate iconicity as one of the main strategies employed whenever signs are grounded from scratch in interaction (Fay et al., 2014; Perlman, Dale, \& Lupyan, 2015; Perlman \& Lupyan, 2018). For example, in studies where participants invent new communication systems using a graphical medium, iconicity serves as a starting point for bootstrapping communication, after which signs become gradually simpler and more symbolic (Caldwell \& Smith, 2012; Garrod, Fay, Lee, Oberlander, \& MacLeod, 2007).

However, if iconicity provides such efficient means to ground a communication system, why do natural languages only display rather subtle aspects of iconicity? And why do we generally observe iconicity to decay over time in favour of more systematic and arbitrary mappings (Garrod et al., 2007; Little, Perlman, \& Eryilmaz, 2017)? As evidenced in, for instance, emerging sign languages (Senghas, Kita, \& Özyürek, 2004; Vos \& Pfau, 2015) home signs (Haviland, 2013; Mylander \& Goldin-Meadow, 1991), and semiotic experimental 
studies (Galantucci, Garrod, \& Roberts, 2012; Tamariz, 2017), signs systems often set off as a set of individual, idiosyncratic mappings to referents (Deacon, 1998). Over time, the sign repertoire evolves and regularities among and relations between individual signs emerge, which eventually give the repertoire properties of a system. As relations among a set of signs stabilize, they provide an alternative means to ground new signs: the meaning of a sign is thus not only constituted by the concrete mapping to a referent, but also by its more abstract relations to other signs in the system. Resulting statistical regularities have been suggested to shift the mnemonic strategy of learners to rely increasingly on the relations among signs (Deacon, 1998) and allow them to quickly categorise newly encountered signs, generalize them and incorporate them into the wider system. Indeed, studies have shown that language-specific statistical differences in word forms (e.g., verb vs. noun morphology) serve as cues that assist category learning (Cassidy \& Kelly, 1991; see Dingemanse et al., 2015 for a review; Monaghan, Chater, \& Christiansen, 2005; Monaghan, Christiansen, \& Chater, 2007). Consequently, signs gradually lose their motivated connection to referents and become increasingly systematic as they come to increasingly depend on their interrelations internally within the communication system.

Thus, while iconicity and systematicity have often been treated as an expression of the same basic pressure of 'motivation' compared to arbitrariness (e.g. Monaghan et al., 2014), they might be better conceived as orthogonal pressures related to different adaptive pressures (see also Dingemanse et al., 2015; Nielsen, 2016).

\subsection{Outline of the study}

The current study was designed to address the circumstances under which systematicity evolves in a novel sign system even if idiosyncratic signs are equally afforded. In many situations both strategies would apply: For instance, if one were to point out a specific person among a crowd of people, one could rely on a single discriminating idiosyncratic trait such as "the person with the funny-looking beard" or "the individual with shiny red shoes": 
However, one could also disambiguate the referent by pointing to a specific combination of more general traits, like gender and job category, as in the example "the female doctor" (as opposed to male doctors). When grounding a novel communication system, what are the conditions that promote the latter systematic (analytic) strategy in contrast to a simple idiosynchratic (iconic, holistic) strategy? That is, when is systematicity more adaptive than encoding single features of referents in one-to-one form-meaning mappings?

Building on the general assumptions of the Linguistic Niche Hypothesis suggesting that systematicity evolves adaptively in response to particular ecological and social affordances, this study sets out to test three complementary hypotheses:

1) Systematicity in communication systems is motivated by regularities in the environment. When new signs evolve under pressure for social coordination and communication about objects and events in the environment, salient relations among those referents provide a semiotic resource, motivating systematic structure among emergent sign forms (Christensen, Fusaroli, \& Tylén, 2016; Lupyan \& Dale, 2016; Tinits, Nölle, \& Hartmann, 2017; Tylén et al., 2013; Winters, Kirby, \& Smith, 2015). Following such predictions, people will be more inclined to selectively systematize those dimensions of their communication system that also appear more structured in the referential environment (Christensen et al., 2016; Nölle, 2015). Returning to the example above, marking the gender of a specific referent would be more adaptive in an environment where the trait is discriminative and thus helps disambiguating between competing referents, that is, in a crowd that consists of both females and males.

2) Systematicity is afforded by ever-changing or unstable environments. Here, we focus on a particular kind of instability, open-endedness. Grounding new signs involves considerable coordination and processing costs for the communicating parties. In "open" environments with novel incoming or changing referents, systematic compositional communication systems provide coordination advantages due to their productivity and thus allow new signs 
to be scaffolded by, or even constructed from, already existing signs (Carr, Smith, Cornish, \& Kirby, 2017; Selten \& Warglien, 2007). Open environments should therefore motivate more attention to abstract regularities that generalize across tokens in the referential context. In other words, we predict systematicity to evolve in response to dynamic environments where novel referents are continuously introduced into the communicative context.

3) Lastly, systematicity is afforded in contexts of displaced communication (when communicating about absent referents). Most studies of emergent communication systems take place in situations where referents are co-present to the interlocutors (e.g. Fay, Arbib, \& Garrod, 2013; Garrod et al., 2007; Scott-Phillips, Kirby, \& Ritchie, 2009). In these situations, less load is put on the working memory of the interlocutors as they can continuously access the referents to incrementally disambiguate the referent target. The need to communicate about episodic content not present in the moment of communication itself provides external pressure on working memory, e.g., by displacing the referential context from the communicative context. This increases the processing load since idiosyncratic features of referents cannot be directly and continuously attended to in the environment but have to be kept in memory. We thus predict more systematicity in contexts of displaced communication as it drastically reduces the working memory load by "chunking" the idiosyncratic properties of many possible referents into more general and economic categories (Christiansen \& Chater, 2016).

In sum, we hypothesize that systematicity is motivated by structural properties of the physical and social environment, and in particular i) that regularities in sign systems reflect regularities in the environment through the principle of functional adaptation, ii) systematicity subserves productivity and is therefore more likely to emerge in an unstable, "open" environment with continuous introduction of new referents, and iii) systematicity will increase in contexts of displaced communication (when communicating about absent references) due to a working memory bottleneck. Furthermore, we predict systematicity to emerge over time during interaction in response to the above factors, and that openness of the environment 
and displacement are likely to interact with time yielding gradually higher levels of systematicity in displaced communication unfolding in open environments.

In order to investigate how the factors described above modulate the relative attraction to competing strategies in the course of stabilizing a novel communication system, we constructed stimuli that would lend themselves to multiple referential solutions. In other words, a target referent in the stimulus set could both be disambiguated using an idiosyncratic iconic or a systematic strategy. In this context, an idiosyncratic strategy would be to use an iconic sign to refer to the referent by attracting attention to a maximally discriminable, unique trait (e.g., a detail that is not shared with any other stimulus item). In contrast, a systematic strategy would entail using combinatorial, analytic forms to draw attention to certain combinations of traits shared across stimulus items and thereby disambiguate the target referent.

We used the "silent gesture" paradigm (Christensen et al., 2016; Fay et al., 2013; Schouwstra \& de Swart, 2014) in which dyads have to solve a referential task by inventing a novel gestural communication system. Experiment 1 tests hypotheses 1 and 2, while experiment 2 tests hypotheses 2 and 3.

\section{Experiment 1: Systematicity is motivated by structural properties of the}

\section{environment}

\subsection{Methods}

\subsubsection{Participants}

27 pairs of participants ( 9 male, 8 female, 10 mixed, mean age 22.9 , SD 5.3) took part in the experiment in return for monetary compensation. Participants were recruited among students at Aarhus University, Denmark. Some of the pairs knew each other in advance. All were native speakers of Danish. 


\subsubsection{Materials}

24 cartoon images of different human characters were created using the freely available online-software bitstrips (Blackstock \& Brown, 2009) and modified for the purpose of the study with the open-source editor Inkscape (The Inkscape Team, 2003). Each character was designed to be individually identifiable from among the full set by either a unique feature (e.g., necklace, glasses, beard etc.) or as a member of three types of categories PROFESSION (e.g., cleric, construction worker, chef), GENDER (male/female) and PET OWNERSHIP (yes/no). These traits were distributed evenly across the full set in such a way that there was one character per combination of categories (e.g., female soldier with a pet; see figure 1 for examples and supplementary material for the full set), leading to a total of four characters (male/female, with/without pet) per profession. In the experimental task, this equally afforded two competing solutions: Using silent gesture, participants could either refer to unique features of the complex stimuli by using iconic gestures (e.g., gesturing glasses) or abstract away from these concrete features and gesture combinations of general categories in a systematic way to disambiguate referents (e.g., male chef without pet). Pseudo-random subsets of 14 stimuli were created for presentation, keeping the following constraints: Each subset included two characters from each of the six job categories, and one additional pair from one of the categories. One of the binary traits (PET, GENDER) was chosen to be the more functionally adaptive trait in the set, that is, it was distributed evenly over the selected meaning space (giving a 50-50 split) and therefore had a higher discriminative value, i.e., helped individuate characters within the same job category (see figure 2). The other, less functionally adaptive trait was distributed 10-4 within the set and thus had a lower discriminative value, both overall and within categories. To control for markedness (e.g., 'female' might be more (conceptually) marked than 'male', and 'with pet' more marked than 'without pet'), the distribution of the less functional trait was counterbalanced across pairs/sets (e.g., sets with 10 males and 4 females vs. sets with 10 females and 4 males). 

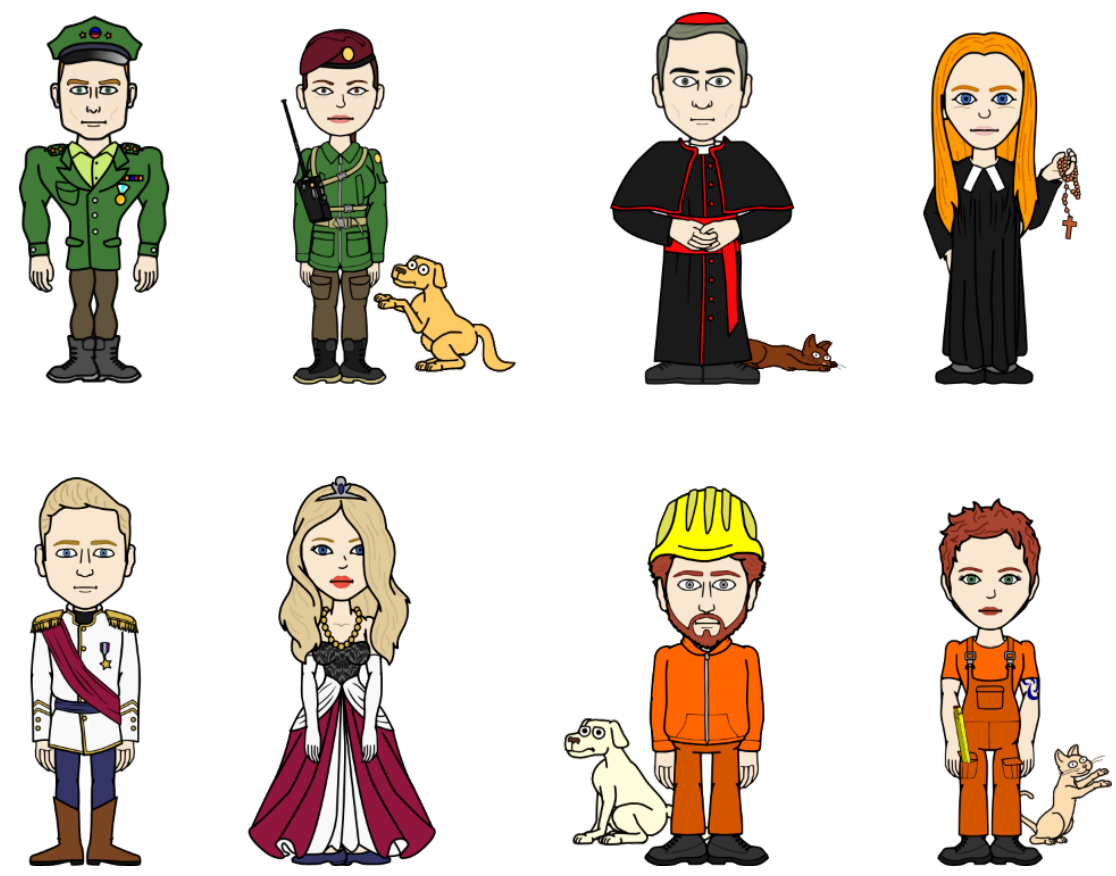

Figure 1: Example Stimuli. Members of the same category share colours, which cannot easily be represented by iconic gestures, but make the categories more salient.

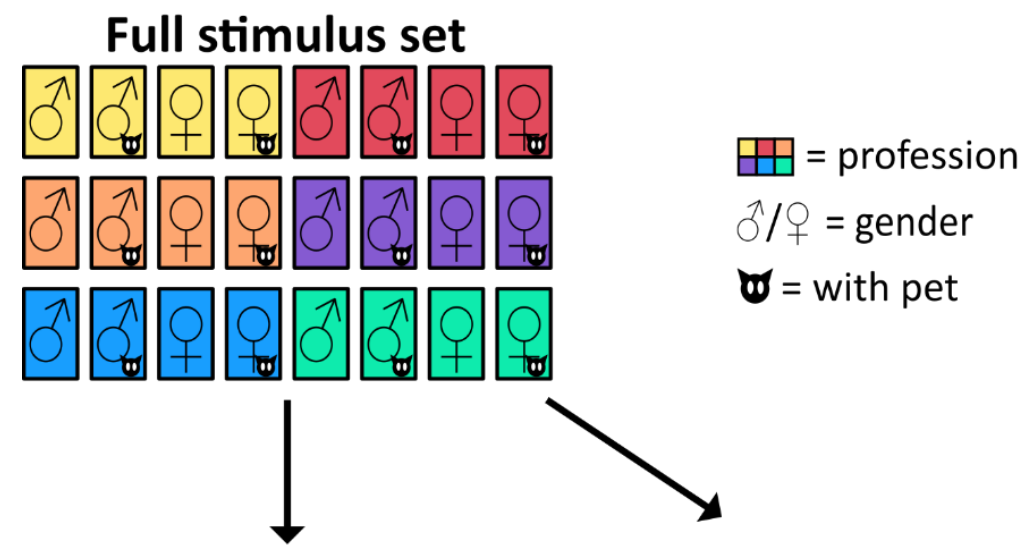

\section{Condition 1}
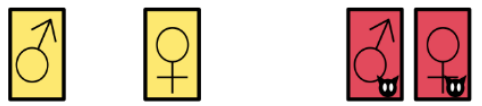

Condition 2

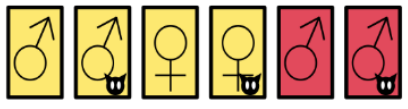

0
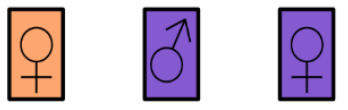

$T_{-1}$
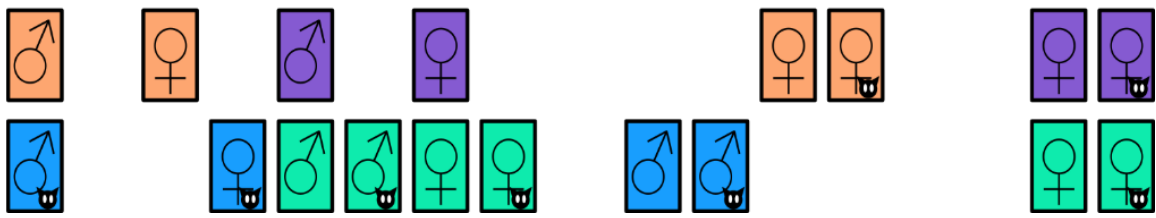

Figure 2: Example trial sets. In condition 1 on the left, the gender trait divides the meaning space in equally sized subsets and is the best discriminating feature within job categories. In condition 2 on the right, the pet trait is more functionally adaptive. Both traits do however occur in both conditions. 


\subsubsection{Design and procedure}

The study employed a two-by-two factorial design with functional adaptivity (high/low) as the first factor and referent environment (open/closed) as the second factor. Functional adaptivity was manipulated within pairs, as one trait (PET/GENDER) was always chosen to be more functionally adaptive than the other within the stimulus set a pair received. The distribution of traits (GENDER more discriminative/PET more discriminative) was counterbalanced across pairs. In addition, "markedness" of the traits was also counterbalanced. For example, in a set in which gender was more functionally adaptive, a large number of stimuli depicted with pet would make the trait PET still more salient compared to a set where most stimuli are depicted without pet.

Pairs were assigned randomly to one of two referential environment conditions: In the 'closed' condition, participants were, on any given trial, presented with one target and 13 foils (i.e. non-target images) drawn from the same set of 14 stimulus items. This was contrasted with the 'open' condition, in which the same targets were still drawn from a stable set of 14 items while foils were sampled from the full set of images and kept changing on every trial, although retaining the distributional properties of the first set. Therefore, the functional adaptivity of traits nevertheless remained stable. This allowed simulating a more 'open' environment, where new referents are constantly introduced, while keeping the demands on communication and memory constant across conditions.

Upon entering the lab, participants were informed that this was a study about the evolution of novel sign languages, and that for this purpose they had to use gestures to communicate about visual stimuli, without talking or making any communicative sounds. They then gave their informed written consent, including permission to video-record their interactions. Further written instructions explaining the details of turn-taking and feedback were given on screen controlled by the stimulus presentation software PsychoPy2 (Peirce, 2007). Partners were seated across from each other at a table, each with a 22-inch (1920 x 1080) monitor 
set up to one side (Figure 3). In each trial, one participant was assigned to be the gesturer, who had to communicate a given stimulus to her partner, and the other to be the guesser, who had to identify that stimulus from the set of 14 stimuli. All 14 stimuli of a set were visible to both the gesturer and the guesser at all times, with a red frame indicating to the gesturer which stimulus she had to communicate. Once the guesser had selected one of the referents from the set by clicking on it with the mouse, auditory feedback was played to indicate success or failure, before moving on to the next trial. Roles were switched after every trial. Even though participants were not explicitly instructed to do so, turn-taking within trials was allowed, meaning that the guesser could ask "questions" (in gestures) or signal understanding. The experiment consisted of four rounds, during each of which each stimulus was communicated once by each pair member (in randomized order), yielding a total of 112 trials per pair. Interactions were recorded with a GoPro Hero 4 camera.
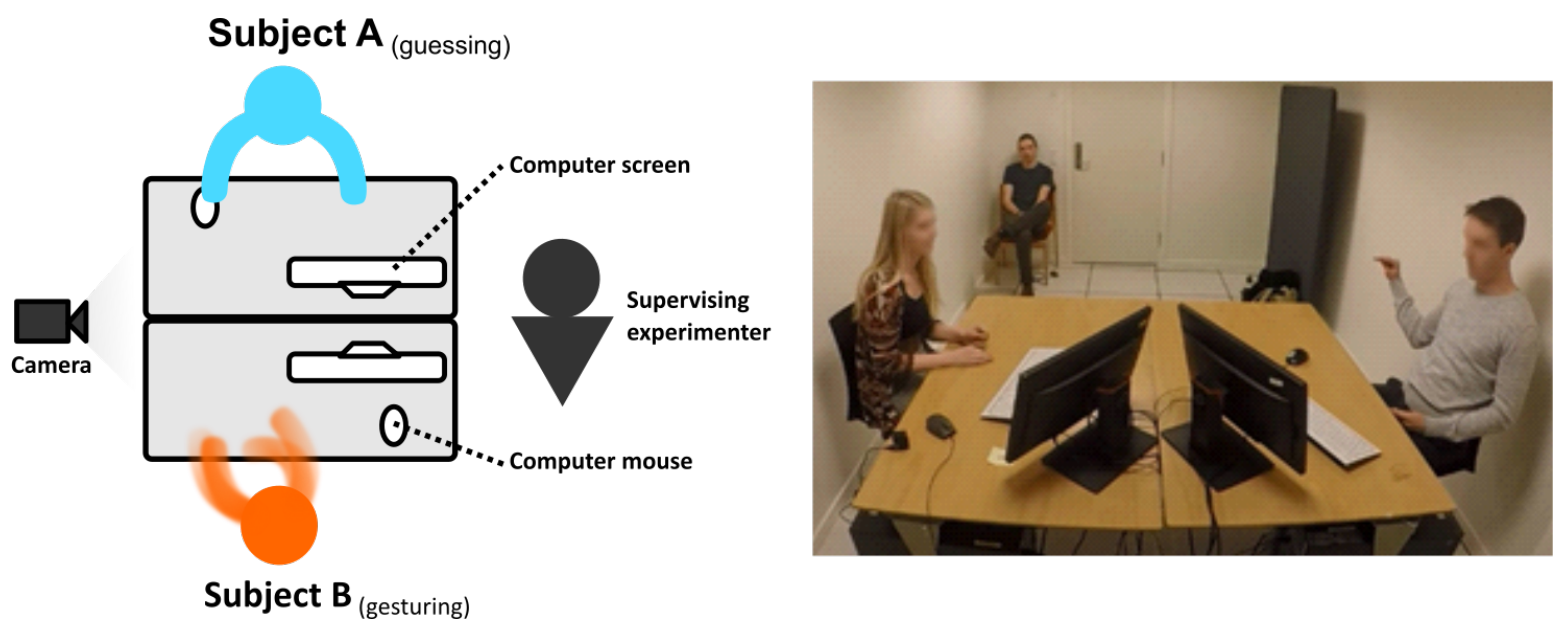

Figure 3: Experimental setup.

\subsubsection{Analysis}

Coding scheme: Gesture strings were analysed on the dyadic level, that is, any gestures produced by either the gesturer or the guesser during a trial were considered to be part of the gesture string for that trial. Individual gestures were classified as systematic by a human coder whenever the same gesture was used to communicate at least two different stimulus items. For example, if the gesturer produces a "stroking" gesture to refer to a pet (e.g., the 
dog next to a soldier), and the same gesture was later used to refer to another pet (e.g., a cat next to a priest), then the gesture is counted as systematic. However, if a new gesture, for instance "paw licking", was used to refer to second stimulus (the cat), it was not counted as systematic, but as idiosyncratic/iconic. The classification only applied looking backward, i.e., from the second referent onwards (and not to any gestures that would only later be used for another referent and thus get systematized). Systematic gestures were further categorized into job, pet and gender gestures - thus allowing for a maximum of 3 (and a minimum of 0 ) instances of systematicity on every trial. The two first authors and one independent coder blind to the hypotheses each coded a third of the data, with 7 percent overlap between each coder pairing (21 percent overlap overall).

Analysis: Intercoder reliability was assessed using percent agreement and unweighted Cohen's Kappa (Cohen, 1960) using the package 'irr' for $R$ (Gamer, Lemon, \& Singh, 2012). Percent accuracy (for guessing the correct stimulus on each trial) was calculated for each condition to confirm the emergence of an efficient communication system, and the development of accuracy over time was estimated using a mixed effects logistic regression model (with random intercepts for subject/pair/condition, coder and stimulus). The number of gestures used (by either the gesturer or the guesser) on each trial was counted and their development over time was analysed analogously to accuracy using a linear mixed effects regression model. The development of the number of gestures per trial was used as a measure of simplification and conventionalization, as it is typically found in experimental studies on emergent communication systems (Caldwell \& Smith, 2012).

In order to test whether hypotheses 1 and 2 were supported by our experimental data, we built a logistic mixed-effects regression model with systematicity (per each feature) as binomial outcome variable and referent environment (open/closed), functional adaptivity (low/high), (z-standardized) trial number (1-112) and markedness (trait marked/unmarked) as predictors. Initially, the maximum random effects structure justified by the design was chosen (Barr, Levy, Scheepers, \& Tily, 2013), excluding random slopes for interactions to 
ensure convergence and avoid overparameterization (Bates, Kliegl, Vasishth, \& Baayen, 2015). This yielded the following equation for the model:

Systematicity functional adaptivity * referent environment + trial number + markedness $+(1$

+ functional adaptivity + trial number $\mid$ subject/team/condition $)+(1+$ functional adaptivity + referent environment + markedness $\mid$ stimulus $)+(1 \mid$ coder $)$

To control for possible confounds of pair familiarity (whether the two partners knew each other in advance) and pair gender (male, female, mixed), we included these factors in a follow-up model and assessed whether i) the model improved its likelihood via BIC-based model comparison (Burnham \& Anderson, 2004), and ii) in that case, whether the previous results held. BIC is an index of generalizability of the results, which calculates the likelihood of the model given the data and penalizes it according to the complexity of the model, thus decreasing the risk of overfitting (Brewer, Butler, \& Cooksley, 2016). It is important to note that BIC-based model comparison does not involve $p$-values and therefore is not as prone to false positive inflation as a statistical significance-based model comparison. All models were implemented with the Ime4 package (Bates, Maechler, Bolker, \& Walker, 2015) in the R environment (R Core Team, 2014).

In order to better assess predicted effects that were non-significant in our model, we calculated the Bayes Factor (Wagenmakers, Lodewyckx, Kuriyal, \& Grasman, 2010) to assess whether the data supported our original hypothesis or whether, to the contrary, the null hypothesis would be a better explanation of our empirical findings. We used the SavageDickey method and weakly sceptical priors for the effects (normal distribution centred at 0 , with a standard deviation of 1). The full analyses scripts and dataset can be viewed at https://osf.io/h5eas/?view_only=8635c0d1001a4de8a4a4a4a42bbe409d. 


\subsection{Results}

\subsubsection{Inter-coder reliability}

Inter-coder reliability was found to be between 94 and 97 percent between the different pairs of coders, corresponding to a Cohen's $x=0.81-0.88$.

\subsubsection{Accuracy and conventionalization}

The overall mean accuracy was high at 98 percent in both conditions, indicating that pairs were communicatively efficient already from the start of the experiment. Accuracy started at a mean of 93 percent on the first trial and increased significantly $(p<.001)$ to over 99 percent on the last trial. By contrast, the number of gestures produced per turn decreased significantly $(p<.001)$ - starting at a mean of 4.3 gestures in the first trial and decreasing to an average of 1.3 gestures by the end of the experiment (after 112 trials) with no difference between conditions ( $p=.04$, see figure 4$)$.

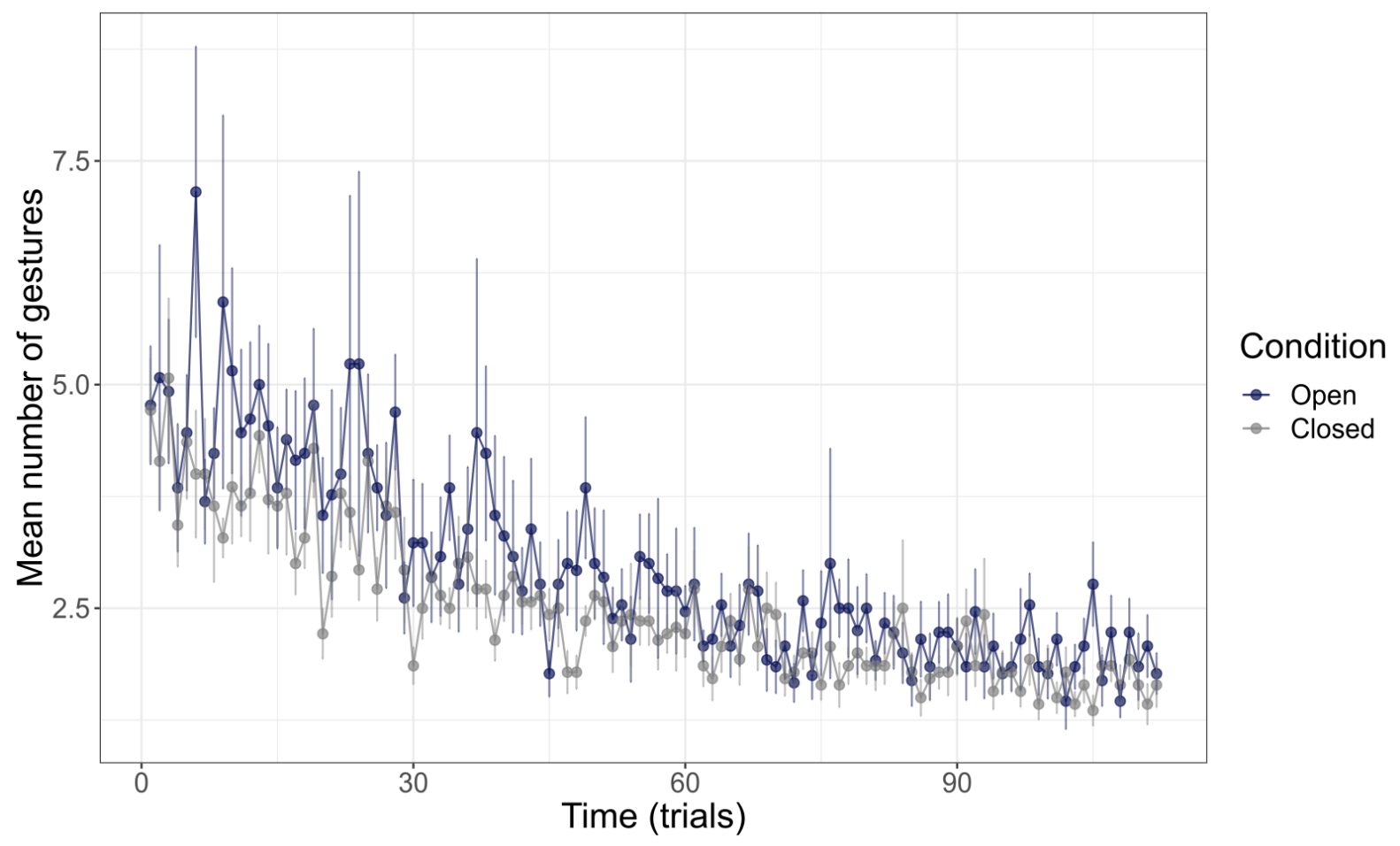

Figure 4: The mean number of gestures per trial in the open and closed environment conditions. 


\subsubsection{Systematicity}

Our model revealed a significant main effect of functional adaptivity $(p<.01)$, as well as a significant min effect of time (trial number) on systematicity $(p<.0001)$. In line with our prediction, traits that were distributed to be highly functionally adaptive over the meaning space were more likely systematized $(14.1 \%$ probability on any given trial/trait) than traits with low functional adaptivity $(2.7 \%)$. Contrary to our prediction, the probability of systematic gestures decreased over time (from $10.0 \%$ to $0.7 \%$ over 112 trials). No significant effects of referent environment (open vs. closed), markedness or interactions between referent environment and functional adaptivity were found in the data. A Bayes Factor analysis yielded inconclusive evidence for all null results. Adding pair familiarity and gender improved the likelihood of the model but did not lead to any changes in the original results. Table 1 presents an overview over the different effects in our model. Figure 5 illustrates the main effect of functional adaptivity.

\begin{tabular}{|l|r|r|l|r|}
\hline measure & $\beta$ & SE & z & BF \\
\hline functional adaptivity (high=1, low=0) & 1.789 & 0.610 & $2.93^{* *}$ & 7.99 \\
refererent environment (open=1, closed=0) & -0.512 & 0.638 & -0.80 & 0.7 \\
trial number (z-standardized) & -0.804 & 0.124 & $-6.49 \quad * * *$ & $>1000$ \\
markedness (marked=1, unmarked=0) & 0.770 & 0.573 & -1.34 & 0.98 \\
functional adaptivity x referent environment & -0.300 & 0.813 & -0.36 & 0.63 \\
\hline
\end{tabular}

Table 1 . Study 1 regression model predicting the amount of systematicity used per trial. Note: ${ }^{*} p \leq$ $.05,{ }^{* *} p \leq .01,{ }^{* * *} p \leq .001$. $\beta$ s represent log odds from the logistic regression model. $R^{2}$ (conditional) $=0.78$; $R_{\text {(marginal) }}^{2}=0.11$, corresponding to a Pearson's coefficient of 33 . 


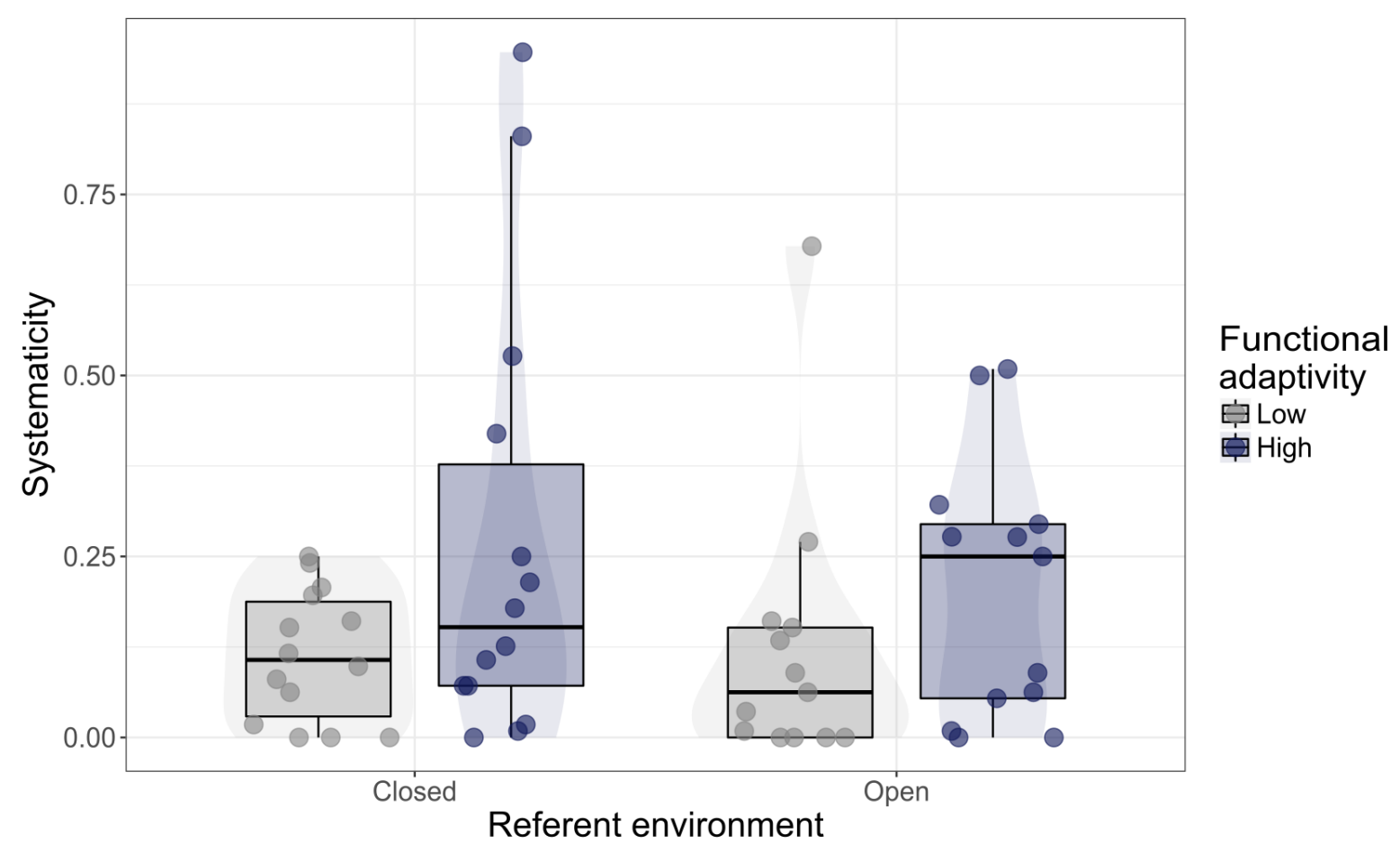

Figure 5: Mean proportion of trials with systematic gestures by functional adaptivity and referent environment. Dots represent proportions of systematicity produced by individual pairs. Note also that the mean is a very imprecise model for this data, as 1) the outcome variable (systematicity) is binary and 2) inter-individual differences are large.

\subsection{Discussion}

Like in previous studies within experimental semiotics, participants in our experiment readily evolved an efficient and expressive communicative sign system to meet the affordances of the referential task in the absence of a previously established communicative conventions (Galantucci, 2009; Galantucci \& Garrod, 2010, 2011; Galantucci et al., 2012). More importantly, in support of hypothesis 1 , we found that structurally distributional properties of the environment, in this case, whether referents can be reliably disambiguated with reference to pet or gender, influence whether or not a trait is picked up and systematized in the sign system. In other words, proto-grammatical features of the emergent gestural communication system seem contingent on structural properties of the referential environment that the participants communicate about. 
We did not find support for the prediction that "open" environments (with foils changing on each trial) afford more systematic signs to emerge. However, we suspect this result to be related to the particular experimental operationalization: As the target stimuli to be communicated still came from a closed set of 14 recurring items, the affordance for systematicity is likely to have been weak. In fact, debriefing revealed that most participants were aware that certain foils were never designated as targets to be communicated, which narrowed down the search space instead of enlarging it.

Lastly, contrary to our predictions, systematicity did not increase, but rather decreased over time during the experiment. Given this initial experimental setup, it thus appears that over repeated interactions communicating a limited set of referents with the same partner, the most efficient strategy is to use highly idiosyncratic, iconic and simplified signs that can be realised in a single gesture.

However, since we identified some limitations in the experimental design that yield the latter observations potentially confounded, we decided to run a second experiment. Here we operationalized the 'openness' of the environment in a more genuine way with new target referents being continuously introduced. Besides, Experiment 2 tests hypothesis 3 , that contexts of displaced communication motivate systematicity due to increased working memory loads.

\section{Experiment 2: Systematicity emerges in displaced interactions and expanding meaning spaces}

\subsection{Methods}

\subsubsection{Participants}

40 pairs of participants $(8 \mathrm{~m}, 13 \mathrm{f}, 19$ mixed, mean age $22.5, \mathrm{SD}=2.1)$ who had not been part of experiment 1 were recruited among students at Aarhus University and took part in the 
experiment in return for monetary compensation. Pair members did not know each other in advance. All were native speakers of Danish.

\subsubsection{Materials, design and procedure}

The same materials and procedure were used as in experiment 1. Eight additional stimuli items (representing two additional job categories: clowns and cave people) were added to increase the set of possible referents. The study employed a two-by-two factorial design with referent environment (open vs. closed) as the first factor and communicative context (displaced/co-present) as the second factor. In contrast to experiment 1, the 'open referent environment' condition in experiment 2 sampled both targets and foils from the full set with no repetition of targets within participants. In each trial, the set consisted of 16 randomly sampled stimuli items. In the closed condition the set remained the same for all trials, while in the open condition it kept changing with the constraint that each set had to contain characters from at least six different professions. This was done to avoid highly imbalanced sets, which could confound communicative behaviour. In the closed referent environment condition, participants played two rounds, during which each pair member communicated each stimulus once. In the open referent environment condition, each stimulus from the full set was communicated once by each pair member, yielding a total of 64 trials in both conditions.

The displacement condition was intended to simulate situations where participants communicate about referents not present in the moment of communication. A trial in the displaced context condition thus proceeded as follows (Figure 6): The gesturer (but not the guesser) had unlimited time to examine the full set and target stimulus (framed in red). When she was ready to proceed, she pressed a button which made the stimuli disappear from the screen while initiating a 3 s countdown, after which she had again unlimited time to produce gestures (in absence of the stimuli). It was then the guesser's turn to indicate when he was ready to make a choice via a button press, again followed by a 3 s countdown and finally the 
full set being displayed to the guesser who then had to make a selection without receiving or producing any further gestures. In the simultaneous communicative context, the procedure was the same as in experiment 1 , with the set being visible to both partners at all times (see figure 6).

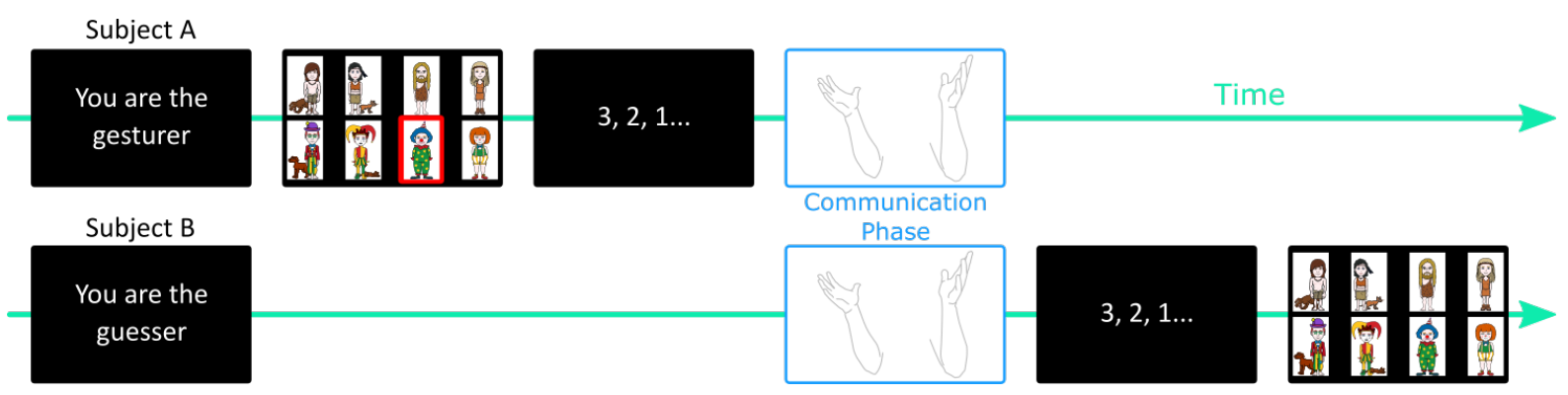

Figure 6: Schematic representation of the procedure for a trial in the 'displaced communicative context' condition.

\subsubsection{Analysis}

The same coding scheme and method for quantitative analysis were used as in experiment 1. An independent coder, blind to the hypothesis, coded 75 percent of the data and JN coded 35 percent (i.e. an overlap of 10 percent). To test hypothesis 2 - 3 we built a factorial logistic regression with systematicity as outcome and displacement and referent environment (including interaction term) and time as predictor variables. As traits were randomly distributed in experiment 1, the kind of trait systematised (systematic job/gender/pet gestures) was included as a random effect in the model. Again, as in experiment 1 , random slopes for interactions were not included for simplicity. Only team gender was added as an additional control variable in the follow-up model, as none of the participants in experiment 2 knew each other in advance.

Systematicity $\sim$ displacement ${ }^{*}$ environment + (displacement + environment $){ }^{*}$ trial number + $(1+$ trial number | team/guesser $)+(1+$ environment + displacement $\mid$ stimulus $)+(1+$ environment + displacement $\mid$ trait $)+(1 \mid$ coder $)$ 
The full scripts and dataset can be viewed at

https://osf.io/h5eas/?view_only=8635c0d1001a4de8a4a4a4a42bbe409d.

\subsection{Results}

\subsubsection{Inter-coder reliability}

Inter-coder reliability was found to be 97 percent, Cohen's $x=0.92$.

\subsubsection{Accuracy and conventionalization}

Again, as in experiment 1 , overall mean accuracy was high, at 95 percent - starting at a mean of 88 percent on the first trial and increasing significantly $(p<.001)$ to over 99 percent on the last trial. Accuracy ranged from 93 to 97 percent in the four conditions, with slightly (but not significantly, $p>.1$ ) different mean accuracy levels in the open (94\%), compared to the closed $(96 \%)$ environment conditions. The number of gestures produced per turn decreased significantly $(p<.001)$ over time - starting at a mean of 5.2 gestures in trial 1 and decreasing to approximately 3.2 gestures. However, there were also condition specific effects with generally more gestures in the open conditions yielding a significant interaction between environment and time ( $p<.0001$, see figure 7 and supplementary material). 


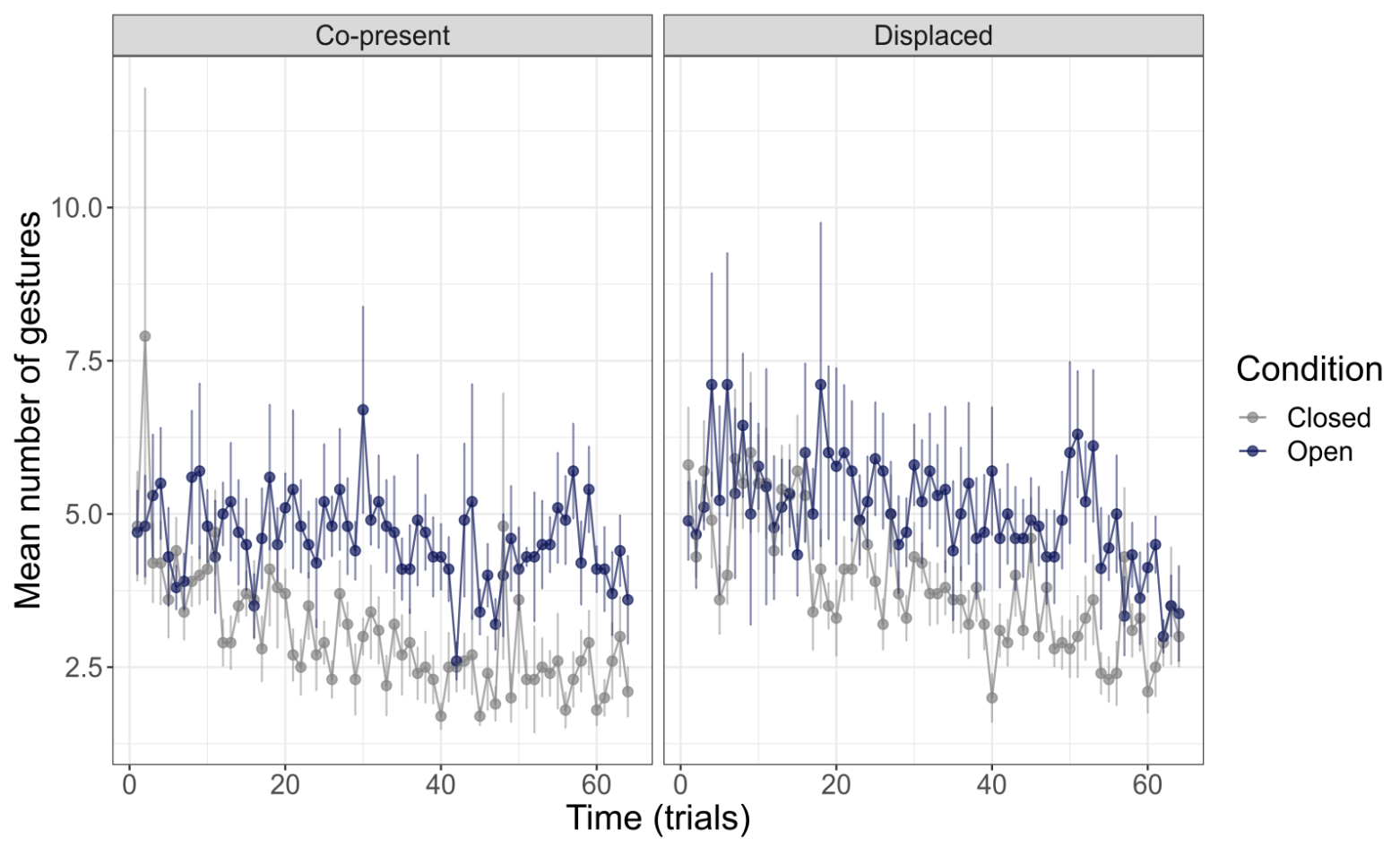

Figure 7: The mean number of gestures over time as a function of the two experimental manipulations.

\subsubsection{Systematicity}

Our model revealed a significant interaction effect between referent environment and trial number $(p<.01)$ as well as a significant main effect of displacement $(p<.05)$. All other effects were non-significant. A Bayes Factor analysis indicated evidence in favour of the null hypothesis for the main effect of trial and the interaction between displacement and trial, and inconclusive evidence in all other cases (including the main effect of displacement). Adding team gender as a covariate did not improve the likelihood of the model given our data. Table 2 presents an overview over the different effects in our predicted model. Figure 8 illustrates the effects of displacement and referent environment. 


\begin{tabular}{|l|c|c|c|c|}
\hline measure & $\boldsymbol{\beta}$ & SE & z & BF \\
\hline displacement (displaced=1, co-present=0) & 0.886 & 0.451 & $1.97 *$ & 1.16 \\
meaning space (open=1, closed=0) & 0.566 & 0.478 & 1.18 & 0.67 \\
trial number (z-standardized) & -0.074 & 0.083 & -0.90 & 0.10 \\
displacement $x$ meaning space & -0.488 & 0.463 & -1.05 & 0.48 \\
displacement $x$ trial number & -0.031 & 0.094 & -0.33 & 0.11 \\
meaning space $x$ trial number & 0.254 & 0.094 & $2.71 * *$ & 2.06 \\
\hline
\end{tabular}

Table 2. Study 2 regression model predicting the amount of systematicity used per trial. Note: ${ }^{*} p \leq$ $.05,{ }^{* *} p \leq .01,{ }^{* * *} p \leq .001$. $\beta$ s represent log odds from the logistic regression model. $R^{2}$ (conditional) $=0.35$; $R_{(\text {marginal) }}^{2}=0.03$, corresponding to a Pearson's coefficient of .17 .

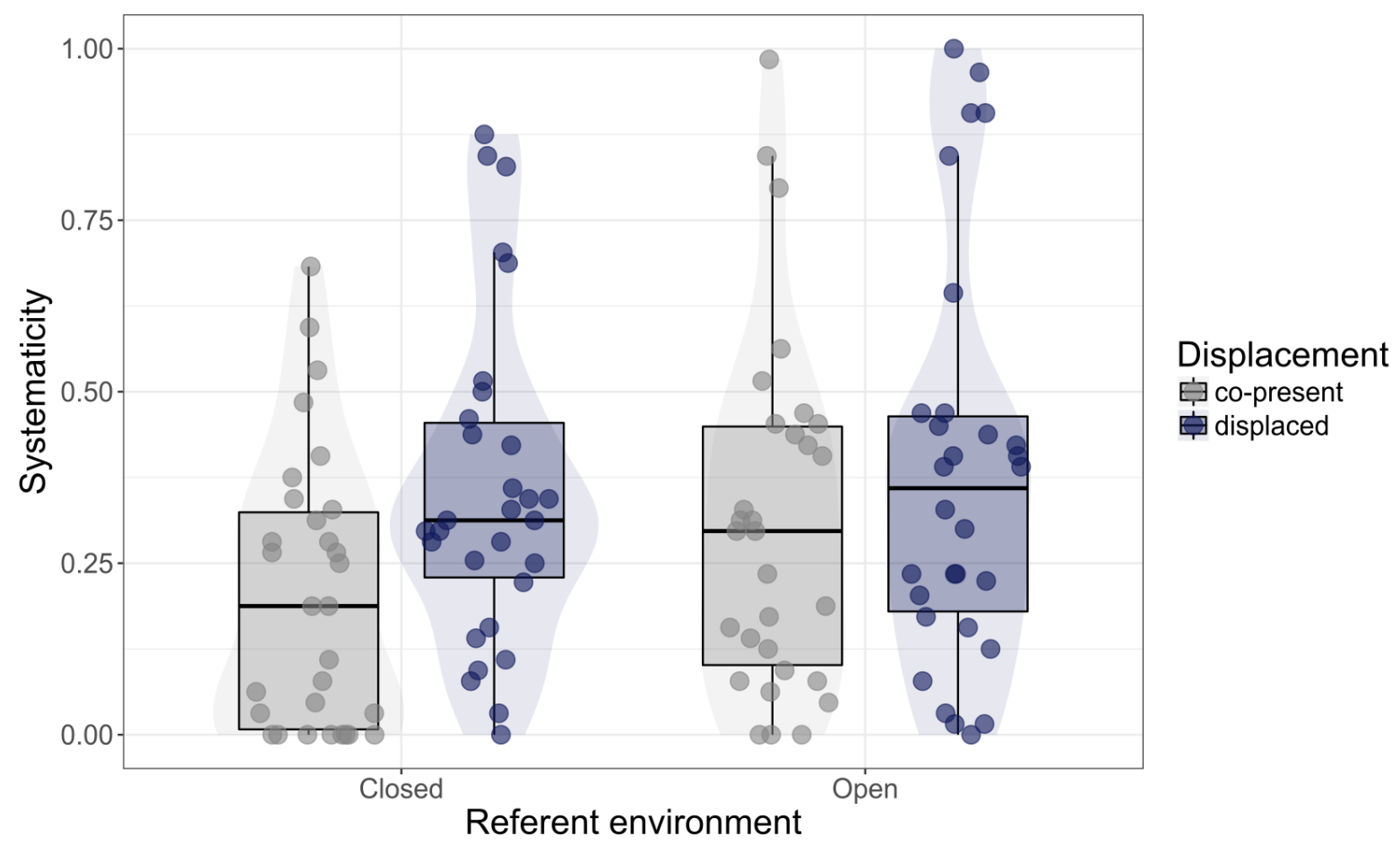

Figure 8: Mean proportion of trials with systematic gestures by environment and displacement condition. Dots represent proportions of systematicity produced by individual pairs. Note also that the 
mean is a very imprecise model for this data, as 1) the outcome variable (systematicity) is binary and 2) inter-individual differences are large.

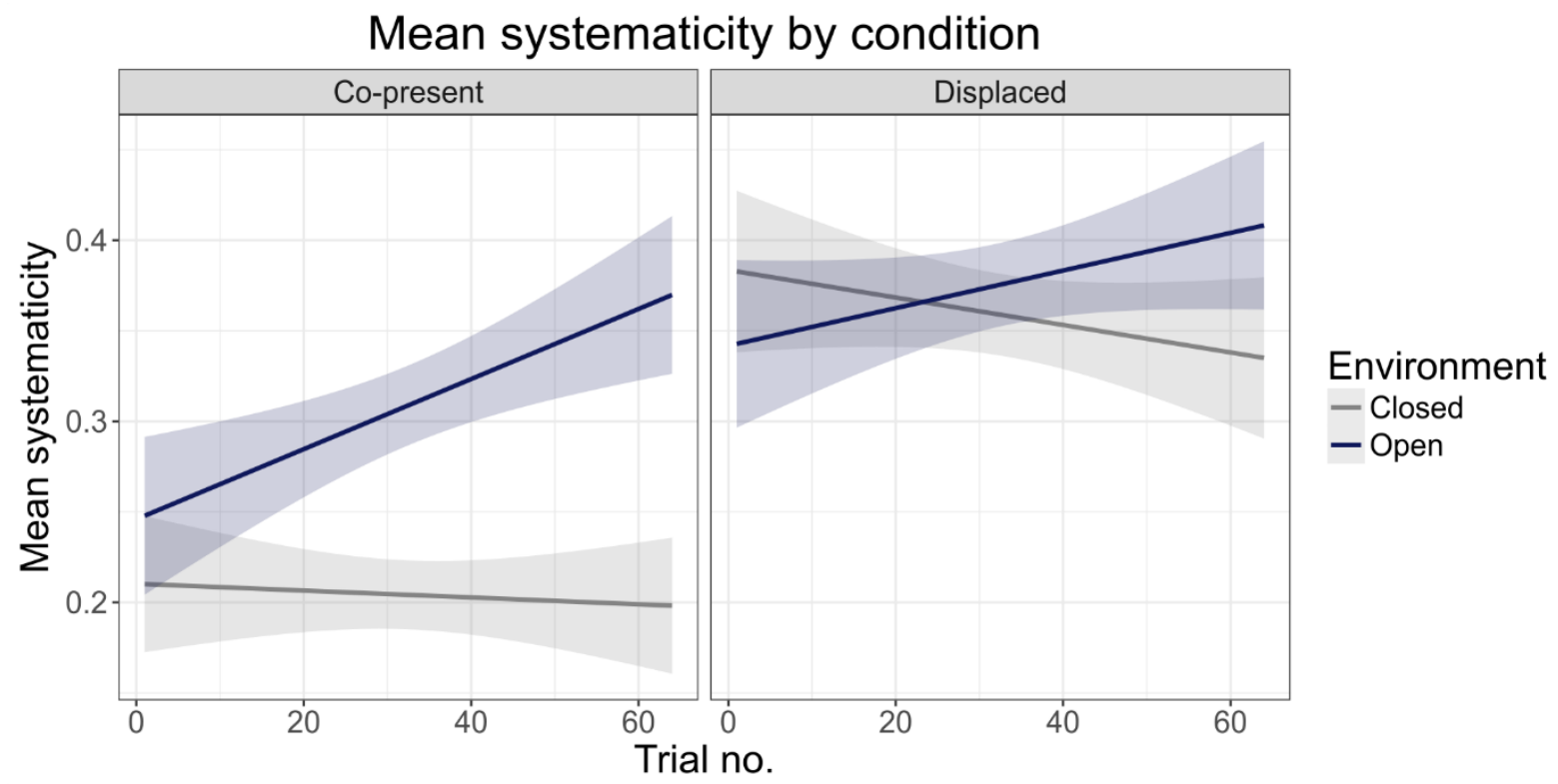

Figure 9: Mean proportion of systematic gestures by trial number. Shaded areas represent betweensubject standard errors, based on individual pair mean proportions. Note also that the mean is a very imprecise model for this data, as 1) the outcome variable (systematicity) is binary and 2) interindividual differences are large.

\subsection{Discussion}

Again, as in experiment 1, participants readily and successfully established an expressive sign system suited to solve the task accurately, and signs became compressed (i.e. simpler) and conventionalized over time. However, the compression was more pronounced in conditions with less systematicity (the closed, co-present condition). With an idiosyncratic strategy, the communication of a referent can potentially be reduced to a single gesture (depicting a single, maximally discriminative feature), while a fully systematic strategy requires the combination of three gestures, one for each of the general features (job, gender and pet). In accordance with hypothesis 2 , we observe stronger propensities to systematize signs in the open-environment condition than in the closed referent environment condition. This effect is modulated by time, as participants experience new referents being continuously added to the meaning space. Similar experimental observations have 
previously been made (Carr et al., 2017; Selten \& Warglien, 2007) supporting the prediction that systematicity evolves adaptively in response to affordances for productivity and flexibility related to unstable, changing or expanding environments.

We also found significant effects of displacement. That is, participants showed higher propensities to use systematic signs when communicating about referents not present in the moment of communication, thus supporting hypothesis 3 . We speculate that such displaced contexts of communication afford participants to abstract and compress information from the immediate visual input into retainable categories more easily sustained in working memory. Unlike open referent environments, the effect of displacement is a main effect and displacement did not interact with time (trial number). In other words, displacement does not lead to a different trajectory of sign evolution over time but shifts the overall probability of systematic signs being used from the beginning of the experiment. This suggests that similarly to learnability in cultural transmission studies (Tamariz, 2017) - displacement poses compressibility demands on emergent sign systems (Christiansen \& Chater, 2016). Against our predictions, we did not find significant interaction effects between displacement and open environments. However, importantly, the evidence for both main effect and the interactions remains inconclusive at best as indicated by Bayes Factor.

\section{General discussion}

\subsection{Systematicity as shaped by the environment}

Here we have presented experimental investigations directly testing the hypothesis that systematicity evolves adaptively in response to external environmental conditions. In two experiments, we investigated the conditions under which new sign systems evolve to show elements of systematicity. A number of studies have suggested systematicity to be contingent mainly on cognitive constraints related to learning in contexts of inter-

generational, "vertical" transmission (Kirby et al., 2008; Kirby et al., 2015; Motamedi, 
Schouwstra, Smith, \& Kirby, 2016; Winters et al., 2015; however, see also Selten \& Warglien, 2007; Theisen, Oberlander, \& Kirby, 2010). In the present study, we show that systematicity can also emerge in contexts of interactive "horizontal" communication in response to particular structural affordances of the environment and the nature of the communicative context. That is, rather than delegating motivation for systematicity alone to internal cognitive biases and constraints, we suggest that systematicity is the adaptive solution to particular 'external' environmental and communicative conditions.

Our findings resonate with a few existing studies on the emergence of systematicity (Motamedi et al., 2016; Theisen et al., 2010). Importantly, in both studies participants' task was to communicate linguistic concepts belonging to broader categories, such as "hair dresser", "hair salon" or "fire station", "fire truck". Not unlike our studies, systematicity was observed to emerge as reuse of sign elements between items belonging to the same categories. Concerns could be raised, however, that the explicit repetition of forms in the linguistic word stimulus (e.g. "fire") across several items directly prime systematic repetitions in participants' communicative responses. Similar concerns can be raised with stimuli used in for instance Kirby et al (2008) and Selten and Warglien (2007) where the highly schematic, combinatorial nature of visual referents constitute a strong prime for systematic responses, while no other solutions are saliently available. In the experiments presented here, categories (JOB, GENDER and PET OWNERSHIP) were more implicit and had to be "discovered" by the participants as a resource for their communicative efforts. In other words, participants had to simultaneously derive perceptual categories (potentially cutting across multiple individual referents) and invent signs to refer to them. Furthermore, our visual stimulus referents supplied participants with competing affordances for idiosyncratic and systematic strategies, which could be argued to yield more ecological validity.

While the emergence of systematicity has been subject of some experimental investigations, less attention has been directed at what aspects of signs are selected for systematicity and what motivates this. In experiment 1 , we manipulated distributional properties of referents 
(whether the referent characters had a pet or was of a specific gender) in order to investigate if the structure of the referent environment affects which semiotic properties are systematized. We found that evenly distributed features with greater discriminative value are more likely to get picked up and systematized in an emerging sign system. This is in line with Christensen and colleagues' (2016) finding that word order in emerging sign systems is motivated by referent event types through principles of "structural iconicity". Experiment 1 , however, goes beyond structural iconicity, as it shows that both the experienced structure of the environment, and the interplay between environment and cognitive efficiency can influence the organization of a communication system: Features with higher informational value (i.e. dividing the search space in a maximally efficient way) are more likely to be systematized, and thus contribute to the communicative efficiency of the sign system.

In experiment 2, we found that the relative stability of the environment influences the development of systematicity over time: Whereas systematicity is unaffected - or even decreases - in stable environments with narrow meaning spaces, open environments with continuously expanding meaning spaces afford for systematic communicative solutions. Combinatorial or compositional signals are more productive and thus are easier to generalize to new referents in the environment than iconic and idiosyncratic form-meaning mappings. In other words, systematicity makes it possible to communicate new referents by reusing and recombining previously established signals thus maintaining the same cognitive economy as in closed meaning spaces. Related observations have been made in an experiment designed to model the costs and benefits of communication (Selten \& Warglien, 2007): When introduced to a larger set of novel referents, pairs of participants who were able to establish a compositional grammar in previous rounds achieved significantly higher gains than those relying on arbitrary mappings or non-compositional grammars (see also Raviv, Meyer, \& Lev-Ari, Under rev.). Importantly, while in Selten and Warglien (2007) and Raviv et al (under rev.) no competing strategies were practically available, the present study shows 
that the productivity advantage remains even when idiosyncratic iconic mappings are possible and communicatively effective (i.e., leading to accurate target identification).

Furthermore, we found systematicity to be afforded in contexts of communicative displacement. Although evidence is inconclusive, participants seemed slightly more inclined to use systematic strategies when they communicated about referents not immediately present at the time of communication. Displacement is widespread in everyday linguistic communication and has been proposed as one of the fundamental design features of language (Hockett, 1960). Bickerton (2009) even speculates that the ability to communicate about absent or even non-existing referents constitutes one of the most profound achievements in the evolution of language. Interestingly, however, it has not previously been manipulated in experimental studies of the evolution of communication systems (however, see Tamariz \& Kirby, 2015 for a non-communicative manipulation of displacement). We hypothesize displacement-related systematicity effects to be contingent on human memory bottlenecks: as representations have to be sustained in memory (in this case only for 3 seconds), they are subject to processes of informational compression and biased reconstruction (Bartlett, 1932; Kirby et al., 2015; Tamariz \& Kirby, 2015). While stochastic idiosyncrasies can be more economic to produce (cf. the lower number of gestures in closed and co-present conditions), they are costlier to encode in memory, since encoding processes are facilitated by structure and rule-bound order (Christiansen \& Chater, 2016). Compression of elements reduces complexity and enables chunking (Chekaf, Cowan, \& Mathy, 2016; Mathy \& Feldman, 2012), which in turn allows encoding elements for subsequent retrieval from long-term working memory (Ericsson \& Kintsch, 1995). Consequently, it is easier to sustain communicative target referents to be communicated as systematic compositional representations than as idiosyncratic tokens. While the potential impact of displacement can be argued to be a 'cognitive' effect (since related to memory), it is brought about by changing the pragmatic context from a situation of communicating about 
co-present referents to one of communicating about referents absent in the moment of communication.

Could the observed effects be driven by other and perhaps simpler factors? In Theisen et al. (2010), systematic responses were observed to often occur when referent stimuli from the same category would appear in immediate succession (e.g. 'school bus' and 'class room'). Curiously, in their study, 21 out of 26 of the initial trials would contain such repetitions of items from the same categories. In these cases, the systematic reuse of items seems motivated by a simple principle of precedence (Garrod \& Pickering, 2004; Pickering \& Garrod, 2004). In our study, the referent stimuli were randomized in ways making these kinds of repetitions quite infrequent. Yet, in order to control for a simple precedence explanation of emergent systematicity behaviour in our experiment, we tested if consecutive occurrences of same JOB, GENDER or PET categories would yield systematic solutions more likely of the corresponding traits than cases without such immediate repetition. In experiment 1 , none of the three features came out significant $(p>.2)$. That is, participants were not more likely to systematize a trait (e.g. a JOB category) when repeated in two consecutive trials. In experiment 2, JOB and PET again came out insignificant, while GENDER was marginally significant $(p=.04)$. However, this seemed driven by later trials. Furthermore, since the number of cases of consecutive trials sharing the same traits were balanced between conditions, a simple precedence factor cannot account for the condition related differences in systematicity reported here.

\subsection{Time}

We hypothesized that systematicity would interact with time, with systematicity increasing over trials. Across experiments, such effects are only observed in the open referent environment condition. In other conditions, systematicity was either stable (displacement condition) or even decreased over time (experiment 1). Similar observations are made by Theisen and colleagues (2010), who also report main effects of systematicity in graphical 
dyadic interaction, with no interactions with time. In the closed referent environment conditions, we rather observe participants through repeated interactions condensing initial elaborative sign ensembles to map minimally discriminative details of the target stimuli, thereby often ending up with a single distinct, non-compositional sign for each referent. This corresponds to general observations in experimental semiotics and shows how, as common ground is build up through repeated "horizontal" interactions among interlocutors, signs become less articulated and simplified (Clark \& Wilkes-Gibbs, 1986; Fay, Garrod, Roberts, \& Swoboda, 2010; Garrod et al., 2007), and thus more "cost-efficient" (i.e. demanding less time and energy to produce), consistent with the proposal of Selten and Warglien (2007). However, along with such processes of compression, signs also tend to gradually shift from iconic towards becoming more symbolic, which makes them less semantically transparent and accessible to newcomers. In other words, such strategies come at the cost of reduced learnability and transparency (Fay \& Ellison, 2013). Vertical transmission (over generations of speakers/signers) on the other hand tends to promote learnability and therefore usually leads to increase in systematicity and regularity (Carr et al., 2017; Kirby et al., 2008; Smith \& Wonnacott, 2010; Verhoef, Kirby, \& de Boer, 2014). In another recent study, Motamedi and colleagues (2016) found that while (horizontal) interaction leads to communicative efficiency, (vertical) transmission over multiple generations poses compressibility demands which leads to systematicity. In their view, only the combination of interaction and transmission leads to the emergence of both communicatively efficient and structured sign systems that resemble real language (Kirby et al., 2015; Regier, Kemp, \& Kay, 2015). Findings from experiment 2 , however, indicate that in contexts of open referent environments, systematicity evolves also gradually in horizontal interaction even if it comes with increased production cost as systematic gestures are more elaborate (e.g. comprising combinations of gestures for GENDER, JOB and PET). 


\subsection{An ecological view of language evolution}

Previous literature has emphasized "inner" cognitive constraints - in particular learner biases amplified through transmission - in explanations of the emergence of systematicity in language (Christiansen \& Chater, 2008; Griffiths \& Kalish, 2007; Kirby et al., 2008; Kirby et al., 2007). In the present experimental approach, we show that systematic structure emerges in response to broader environmental and contextual affordances. While cognitive processes and bottlenecks are certainly involved, the biases giving more specific, directional shape to the evolution of communication systems need not be delegated to latent inner (and thus innate) factors. Outside laboratories, environments are generally open, large and unstable (see also Carr et al., 2017), with natural, cultural and social changes affording flexibility on all levels of sign use (discourse, lexicogrammar, phonetics). Furthermore, contexts of, for instance, collective foraging could possibly have presented strong affordances for displaced communication that again could have shaped our communicative practices towards becoming more systematic (Bickerton, 2009). The morphological features selected for systematicity seem to be contingent on the semiotic potentials of the surrounding environment, that is, the structures stabilizing in evolving communication systems are not independent of the structural organization of the environment that we communicate about (as posited, e.g., by proponents of generative grammar, Chomsky, 1986; Hauser et al., 2002; Pinker, 1994). For instance, if gender provides itself as a reliable trait that can be exploited as semiotic resource for disambiguating referents, we are likely to see this trait getting grammaticalized.

A broader implication of this ecological contextualization of language evolution is that rather than merely presupposing language to be the product of a biological restructuring of the brain (Berwick \& Chomsky, 2015), or an adaptation to fit the architecture of the biological brain (Christiansen \& Chater, 2008), it reflects adaptive cognitive strategies responding to external affordances and pressures manifest in the social or physical environment (Everett et al., 2015; Lupyan \& Dale, 2010, 2016). 


\section{Conclusions}

In two experiments, we tested the hypothesis that systematicity in language evolves adaptively in response to external environmental conditions. Other experimental studies have suggested individual learning mechanisms and cognitive biases related to intergenerational transmission as the major driving force behind the emergence of systematic structure (Kirby et al., 2015; Motamedi et al., 2016; Tamariz \& Kirby, 2016). The experiments presented above extend these findings showing that systematicity emerges also in contexts of horizontal interaction in response to aspects of the communicative situation itself, even in the presence of competing iconic solutions observed to be effective in several previous experimental studies (Fay et al., 2013; Garrod et al., 2007; Perlman et al., 2015).

We have shown that the selection of traits to be systematized depends on their functional adaptivity in the given environment, leading to co-emergence of systematic categories and signals to encode them. In addition, environmental factors also affect the propagation of systematicity in emerging communication systems to the extent that they pose informational bottlenecks. Systematicity is thus more adaptive in an unstable, changing and open environment where analytic forms give flexibility and productivity to communicate about novel referents.

Furthermore, displacement of referents from the communicative context could exert working memory pressures making systematic, compressible structure more favourable. Although displacement is a fundamental property of language (Hockett, 1960), it has been largely neglected in laboratory studies investigating cultural language evolution that have rather emphasized learning. Manipulation of displacement in artificial language learning tasks thus presents itself as a promising avenue for further research in the field. 


\section{Acknowledgements}

We would like to thank the Cognition and Behavior Lab at Aarhus BSS for facilities and equipment as well as Sergio Gonzales De La Higuera Rojo and Caroline Kildahl for coding of the video material. This work was funded by a Seed Grant of the Interactive Minds Centre as well as funding from the School of Communication and Culture, Aarhus University.

\section{Declarations of interest: none}

\section{Appendix A. Supplementary material}

All datasets, analysis scripts and stimuli material can be accessed at

https://osf.io/h5eas/?view_only=8635c0d1001a4de8a4a4a4a42bbe409d.

\section{References}

Barr, D. J., Levy, R., Scheepers, C., \& Tily, H. J. (2013). Random effects structure for confirmatory hypothesis testing: Keep it maximal. Journal of memory and language, 68(3), 255-278.

Bartlett, F. C. (1932). Remembering: A Study in Experimental and Social Psyhology. Cambridge: Cambridge University Press.

Bates, D., Kliegl, R., Vasishth, S., \& Baayen, H. (2015). Parsimonious mixed models. arXiv preprint arXiv:1506.04967.

Bates, D., Maechler, M., Bolker, B., \& Walker, S. (2015). Fitting Linear Mixed-Effects Models Using Ime4. Journal of Statistical Software, 67(1), 1-48.

Bergen, B. K. (2004). The psychological reality of phonaesthemes. Language, 80(2), 290311.

Berwick, R. C., \& Chomsky, N. (2015). Why only us: Language and evolution: MIT press.

Bickerton, D. (2009). Adam's tongue: how humans made language, how language made humans: Hill and Wang.

Blackstock, J., \& Brown, J. (2009). Bitstrips. Toronto, Canada.

Brewer, M. J., Butler, A., \& Cooksley, S. L. (2016). The relative performance of AIC, AICC and $\mathrm{BIC}$ in the presence of unobserved heterogeneity. Methods in Ecology and Evolution, 7(6), 679-692.

Brighton, H. (2002). Compositional syntax from cultural transmission. Artificial Life, 8(1), 2554.

Burnham, K. P., \& Anderson, D. R. (2004). Multimodel inference: understanding AIC and BIC in model selection. Sociological methods \& research, 33(2), 261-304.

Caldwell, C. A., \& Smith, K. (2012). Cultural evolution and perpetuation of arbitrary communicative conventions in experimental microsocieties. PloS one, 7(8), e43807.

Carr, J. W., Smith, K., Cornish, H., \& Kirby, S. (2017). The Cultural Evolution of Structured Languages in an Open-Ended, Continuous World. Cognitive science, 41(4), 892-923.

Cassidy, K. W., \& Kelly, M. H. (1991). Phonological information for grammatical category assignments. Journal of Memory and Language, 30(3), 348-369. 
Chekaf, M., Cowan, N., \& Mathy, F. (2016). Chunk formation in immediate memory and how it relates to data compression. Cognition, 155, 96-107.

Chomsky, N. (1986). Knowledge of Language : Its Nature, Origin, and Use. New York: Praeger.

Christensen, P., Fusaroli, R., \& Tylén, K. (2016). Environmental constraints shaping constituent order in emerging communication systems: Structural iconicity, interactive alignment and conventionalization. Cognition, 146, 67-80.

Christiansen, M., \& Chater, N. (2008). Language as shaped by the brain. Behavioral and Brain Sciences, 31(05), 1-70.

Christiansen, M. H., \& Chater, N. (2016). The Now-or-Never bottleneck: A fundamental constraint on language. Behavioral and Brain Sciences, 39.

Clark, H. H., \& Wilkes-Gibbs, D. (1986). Referring as a collaborative process. Cognition, 22(1), 1-39.

Cohen, J. (1960). A Coefficient of Agreement for Nominal Scales. Educational and Psychological Measurement, 20(1), 37-46.

Dale, R., \& Lupyan, G. (2010). Squiggle: Large-Scale Social Emergence of Simple Symbols. Evolution of Language, Proceedings, 391-+.

Deacon, T. W. (1998). The symbolic species: The co-evolution of language and the brain: WW Norton \& Company.

Dediu, D., Janssen, R., \& Moisik, S. R. (2017). Language is not isolated from its wider environment: Vocal tract influences on the evolution of speech and language. Language \& Communication, 54, 9-20.

Dediu, D., \& Ladd, D. R. (2007). Linguistic tone is related to the population frequency of the adaptive haplogroups of two brain size genes, ASPM and Microcephalin.

Proceedings of the National Academy of Sciences of the United States of America, 104(26), 10944-10949.

Dingemanse, M. (2012). Advances in the cross-linguistic study of ideophones. Language and Linguistics compass, 6(10), 654-672.

Dingemanse, M., Blasi, D. E., Lupyan, G., Christiansen, M. H., \& Monaghan, P. (2015). Arbitrariness, Iconicity, and Systematicity in Language. Trends Cogn Sci, 19(10), 603-615.

Ericsson, K. A., \& Kintsch, W. (1995). Long-term working memory. Psychological Review, 102(2), 211-245.

Everett, C., Blasi, D. E., \& Roberts, S. G. (2015). Climate, vocal folds, and tonal languages: Connecting the physiological and geographic dots. Proceedings of the National Academy of Sciences of the United States of America, 112(5), 1322-1327.

Everett, C., Blasi, D. E., \& Roberts, S. G. (2016). Language evolution and climate: the case of desiccation and tone. Journal of Language Evolution, 1(1), 33-46.

Fay, N., Arbib, M., \& Garrod, S. (2013). How to bootstrap a human communication system. Cogn Sci, 37(7), 1356-1367.

Fay, N., \& Ellison, T. M. (2013). The cultural evolution of human communication systems in different sized populations: usability trumps learnability. PloS one, 8(8), e71781.

Fay, N., Ellison, T. M., \& Garrod, S. (2014). Iconicity: from sign to system in human communication and language. Pragmatics \& Cognition, 22(2), 244-263.

Fay, N., Garrod, S., Roberts, L., \& Swoboda, N. (2010). The interactive evolution of human communication systems. Cogn Sci, 34(3), 351-386.

Galantucci, B. (2009). Experimental semiotics: a new approach for studying communication as a form of joint action. Topics in Cognitive Science, 1(2), 393-410.

Galantucci, B., \& Garrod, S. (2010). Experimental semiotics: A new approach for studying the emergence and the evolution of human communication. Interaction Studies: Social Behaviour and Communication in Biological and Artificial Systems.

Galantucci, B., \& Garrod, S. (2011). Experimental semiotics: a review. Frontiers in human neuroscience, 5, 11.

Galantucci, B., Garrod, S., \& Roberts, G. (2012). Experimental semiotics. Language and Linguistics Compass, 6(8), 477-493. 
Gamer, M., Lemon, J., \& Singh, I. (2012). irr: Various Coefficients of Interrater Reliability and Agreement. $R$ package version 0.84 .

Garrod, S., Fay, N., Lee, J., Oberlander, J., \& MacLeod, T. (2007). Foundations of representation: where might graphical symbol systems come from? Cognitive Science, 31(6), 961-987.

Garrod, S., \& Pickering, M. J. (2004). Why is conversation so easy? Trends in Cognitive Sciences, 8(1), 8-11.

Griffiths, T. L., \& Kalish, M. L. (2007). Language evolution by iterated learning with Bayesian agents. Cognitive science, 31(3), 441-480.

Harnad, S. (1990). The Symbol Grounding Problem. Physica D, 42(1-3), 335-346.

Hauser, M. D., Chomsky, N., \& Fitch, W. T. (2002). The faculty of language: what is it, who has it, and how did it evolve? Science, 298(5598), 1569-1579.

Haviland, J. B. (2013). The emerging grammar of nouns in a first generation sign language: specification, iconicity, and syntax. Gesture, 13(3), 309-353.

Hockett, C. F. (1960). The Origin of Speech. Scientific American, 203(3), 88-96.

Imai, M., \& Kita, S. (2014). The sound symbolism bootstrapping hypothesis for language acquisition and language evolution. Philosophical Transactions of the Royal Society B-Biological Sciences, 369(1651).

Imai, M., Kita, S., Nagumo, M., \& Okada, H. (2008). Sound symbolism facilitates early verb learning. Cognition, 109(1), 54-65.

Kirby, S., Cornish, H., \& Smith, K. (2008). Cumulative cultural evolution in the laboratory: an experimental approach to the origins of structure in human language. Proc Natl Acad Sci U S A, 105(31), 10681-10686.

Kirby, S., Dowman, M., \& Griffiths, T. L. (2007). Innateness and culture in the evolution of language. Proc Natl Acad Sci U S A, 104(12), 5241-5245.

Kirby, S., Griffiths, T., \& Smith, K. (2014). Iterated learning and the evolution of language. Curr Opin Neurobiol, 28, 108-114.

Kirby, S., Tamariz, M., Cornish, H., \& Smith, K. (2015). Compression and communication in the cultural evolution of linguistic structure. Cognition, 141, 87-102.

Lindsey, D. T., \& Brown, A. M. (2002). Color naming and the phototoxic effects of sunlight on the eye. Psychological Science, 13(6), 506-512.

Little, H., Perlman, M., \& Eryilmaz, K. (2017). Repeated interactions can lead to more iconic signals. Paper presented at the the 39th Annual Conference of the Cognitive Science Society (CogSci 2017).

Lockwood, G., Dingemanse, M., \& Hagoort, P. (2016). Sound-Symbolism Boosts Novel Word Learning. Journal of Experimental Psychology-Learning Memory and Cognition, 42(8), 1274-1281.

Lupyan, G., \& Dale, R. (2010). Language Structure Is Partly Determined by Social Structure. Plos One, 5(1).

Lupyan, G., \& Dale, R. (2016). Why Are There Different Languages? The Role of Adaptation in Linguistic Diversity. Trends in Cognitive Sciences, 20(9), 649-660.

Mathy, F., \& Feldman, J. (2012). What's magic about magic numbers? Chunking and data compression in short-term memory. Cognition, 122(3), 346-362.

Monaghan, P., Chater, N., \& Christiansen, M. H. (2005). The differential role of phonological and distributional cues in grammatical categorisation. Cognition, 96(2), 143-182.

Monaghan, P., Christiansen, M. H., \& Chater, N. (2007). The phonological-distributional coherence hypothesis: Cross-linguistic evidence in language acquisition. Cognitive psychology, 55(4), 259-305.

Monaghan, P., Mattock, K., \& Walker, P. (2012). The Role of Sound Symbolism in Language Learning. Journal of Experimental Psychology-Learning Memory and Cognition, 38(5), 1152-1164.

Monaghan, P., Shillcock, R. C., Christiansen, M. H., \& Kirby, S. (2014). How arbitrary is language? Philosophical Transactions of the Royal Society B-Biological Sciences, 369(1651). 
Motamedi, Y., Schouwstra, M., Smith, K., \& Kirby, S. (2016). Linguistic Structure Emerges In The Cultural Evolution Of Artificial Sign Languages. Paper presented at the The Evolution of Language: Proceedings of the 11th International Conference (EVOLANG11).

Mylander, C., \& Goldin-Meadow, S. (1991). Home sign systems in deaf children: The development of morphology without a conventional language model. Theoretical issues in sign language research, 2, 41-63.

Nielsen, A. (2016). Systematicity, motivatedness, and the structure of the lexicon. University of Edinburgh.

Nölle, J. (2015). Environmental Constraints on Cultural Conventions: A Case Study of Linguistic Coordination in Joint Problem Solving. Aarhus University.

Peirce, C. S. (1931). Collected papers of Charles Sanders Peirce. Cambridge,: Harvard University Press.

Peirce, J. W. (2007). PsychoPy--Psychophysics software in Python. J Neurosci Methods, 162(1-2), 8-13.

Perlman, M., Dale, R., \& Lupyan, G. (2015). Iconicity can ground the creation of vocal symbols. Royal Society open science, 2(8), 150152.

Perlman, M., Fusaroli, R., Fein, D., \& Naigles, L. (2017). The Use of Iconic Words in Early Child-Parent Interactions. Paper presented at the the 39th Annual Conference of the Cognitive Science Society (CogSci 2017).

Perlman, M., \& Lupyan, G. (2018). People Can Create Iconic Vocalizations to Communicate Various Meanings to Naïve Listeners. Scientific Reports, 8(2634), 148841.

Perniss, P., Thompson, R. L., \& Vigliocco, G. (2010). Iconicity as a general property of language: evidence from spoken and signed languages. Frontiers in psychology, 1.

Perry, L. K., Perlman, M., \& Lupyan, G. (2015). Iconicity in English and Spanish and Its Relation to Lexical Category and Age of Acquisition. Plos One, 10(9).

Pickering, M. J., \& Garrod, S. (2004). Toward a mechanistic psychology of dialogue. Behavioral and Brain Sciences, 27(2), 169-190; discussion 190-226.

Pinker, S. (1994). The Language Instinct. Harmondsworth: Allen Lane.

R Core Team. (2014). R: A language and environment for statistical computing. Vienna, Austria: R Foundation for Statistical Computing.

Raviv, L., Meyer, A., \& Lev-Ari, S. (Under rev.). Compositional structure can emerge without generational transmission.

Regier, T., Carstensen, A., \& Kemp, C. (2016). Languages Support Efficient Communication about the Environment: Words for Snow Revisited. PLoS One, 11(4), e0151138.

Regier, T., Kemp, C., \& Kay, P. (2015). Word meanings across languages support efficient communication. The handbook of language emergence, 237-263.

Saussure, F. d. (1959). Course in general linguistics. New York,: Philosophical Library.

Schouwstra, M., \& de Swart, H. (2014). The semantic origins of word order. Cognition, 131(3), 431-436.

Scott-Phillips, T. C., Kirby, S., \& Ritchie, G. R. (2009). Signalling signalhood and the emergence of communication. Cognition, 113(2), 226-233.

Selten, R., \& Warglien, M. (2007). The emergence of simple languages in an experimental coordination game. Proceedings of the National Academy of Sciences, 104(18), 7361-7366.

Senghas, A., Kita, S., \& Özyürek, A. (2004). Children creating core properties of language: Evidence from an emerging sign language in Nicaragua. Science, 305(5691), 17791782.

Smith, K., Brighton, H., \& Kirby, S. (2003). Complex systems in language evolution: The cultural emergence of compositional structure. Advances in Complex Systems, 6(4), 537-558.

Smith, K., \& Wonnacott, E. (2010). Eliminating unpredictable variation through iterated learning. Cognition, 116(3), 444-449.

Tamariz, M. (2017). Experimental studies on the cultural evolution of language. Annual Review of Linguistics, 3, 389-407. 
Tamariz, M., \& Kirby, S. (2015). Culture: copying, compression, and conventionality. Cognitive science, 39(1), 171-183.

Tamariz, M., \& Kirby, S. (2016). The cultural evolution of language. Current Opinion in Psychology, 8, 37-43.

The Inkscape Team. (2003). Incscape.

Theisen, C. A., Oberlander, J., \& Kirby, S. (2010). Systematicity and arbitrariness in novel communication systems. Interaction Studies, 11(1), 14-32.

Tinits, P., Nölle, J., \& Hartmann, S. (2017). Usage context influences the evolution of overspecification in iterated learning. Journal of Language Evolution.

Tylén, K., Fusaroli, R., Bundgaard, P. F., \& Østergaard, S. (2013). Making sense together: A dynamical account of linguistic meaning-making. Semiotica, 2013(194), 39-62.

Verhoef, T., Kirby, S., \& de Boer, B. (2014). Emergence of combinatorial structure and economy through iterated learning with continuous acoustic signals. Journal of Phonetics, 43, 57-68.

Vos, C. d., \& Pfau, R. (2015). Sign language typology: the contribution of rural sign languages. Annu. Rev. Linguist., 1(1), 265-288.

Wagenmakers, E.-J., Lodewyckx, T., Kuriyal, H., \& Grasman, R. (2010). Bayesian hypothesis testing for psychologists: A tutorial on the Savage-Dickey method. Cognitive psychology, 60(3), 158-189.

Winters, J., Kirby, S., \& Smith, K. (2015). Languages adapt to their contextual niche. Language and Cognition, 7(3), 415-449. 